\title{
Thermalization in the Two-Body Random Ensemble
}

\author{
V. K. B. Kota ${ }^{1}$, A. Relaño ${ }^{2}$, J. Retamosa ${ }^{3}$, Manan Vyas ${ }^{1}$ \\ ${ }^{1}$ Physical Research Laboratory, Ahmedabad 380 009, India \\ ${ }^{2}$ Instituto de Estructura de la Materia, IEM-CSIC Serrano, 123, 28006-Madrid. \\ Spain \\ ${ }^{3}$ Grupo de Física Nuclear, Departamento de Física Atómica, Molecular y Nuclear, \\ Universidad Complutense de Madrid, E-28040 Madrid, Spain
}

\begin{abstract}
Using the ergodicity principle for the expectation values of several types of observables, we investigate the thermalization process in isolated fermionic systems. These are described by the two-body random ensemble, which is a paradigmatic model to study quantum chaos and specially the dynamical transition from integrability to chaos. By means of exact diagonalizations we analyze the relevance of the eigenstate thermalization hypothesis as well as the influence of other factors, like the energy and structure of the initial state, or the dimension of the Hilbert space. We also obtain analytical expressions linking the degree of thermalization for a given observable with the so-called number of principal components for transition strengths originated at a given energy, with the dimensions of the whole Hilbert space and microcanonical energy shell, and with the correlations generated by the observable. As the strength of the residual interaction is increased an order-to-chaos transition takes place, and we show that the onset of Wigner spectral fluctuations, which is the standard signature of chaos, is not sufficient to guarantee thermalization in finite systems. When all the signatures of chaos are fulfilled, including the quasi complete delocalization of eigenfunctions, the eigenstate thermalization hypothesis is the mechanism responsible for the thermalization of certain types of observables, such as (linear combinations of) occupancies and strength function operators. Our results also suggest that fully chaotic systems will thermalize relative to most observables in the thermodynamic limit.
\end{abstract}

Keywords: Connections between chaos and statistical physics, Matrix models, Quantum chaos 


\section{Introduction}

The success of thermodynamics is based on the fact that the state of a macroscopic system behaves as it were independent of the microscopic details of the system, and thus it can be determined from a few universal laws. In classical isolated systems this thermodynamic universality can be explained satisfactorily if one assumes that the dynamics is ergodic and mixing. These properties define classical deterministic chaos [1] and lead to the equiprobability of equal-volume regions of the available phase space, which is the starting point of the microcanonical ensemble formulation. This implies that, on average, the state of the system is independent of the initial conditions as well as independent of the measurement time. It is thus generally accepted that the system equilibrates into a thermal state, described by the microcanonical ensemble, when its dynamical regime is ergodic. On the other hand thermalization is expected to be inhibited for integrable or quasi integrable systems. In these systems (almost) every trajectory in phase space lies in one of the so-called invariant tori, which foliate the phase space. Therefore, being restricted the trajectories to these structures, the equiprobability equal-volume regions of the available phase space does not hold and the system will not thermalize, at least in the sense explained above.

In recent years the study of the equilibration and thermalization mechanisms in isolated quantum systems has attracted a great interest partly because the non-equilibrium dynamics, after an external perturbation is applied, has become experimentally accessible for ultra-cold quantum gases [2, 3] and electrons in solids [4]. The technology makes it possible to induce sharp changes in the parameters controlling the system and then observe the subsequent time evolution, which is essentially unitary because on short and intermediate time scales the perturbed system is almost isolated from the environment. Thus, one can experimentally study if an isolated system equilibrates after a sharp perturbation and in this case whether it thermalizes or retains memory of the initial conditions. Experimental studies of the non-equilibrium dynamics in one-dimensional ultra-cold Bose gases have given, up to the moment, contradictory results [5, 6]

From a theoretical point of view, these questions have been addressed using different methods and points of view. The results from classical mechanics can not be translated directly into quantum mechanics. On one hand the concept of quantum integrability is not well defined, though it is generally considered as synonym for exact solvability [7, 8]. On the other hand the unitary time evolution of quantum states leaves no room for a dynamical regime similar to deterministic chaos. Actually, the name quantum chaos stands for the different type of signatures that certain quantum systems exhibit depending on the large time scale behavior of their classical analogues [9, 10]. These signatures appear on the statistical behavior of eigenenergies and eigenfunctions and is not clear at all how they can influence the evolution of the system on large-time scales.

The generally accepted assumption that integrable systems do not thermalize is corroborated in several models [11, 12, 13, 14, 15], where the non-equilibrium regime 
extends over large time scales or where a long-time steady state with non standard thermal properties is reached. The approach to thermal equilibrium of generic systems has been studied by several authors. It has been proven that almost any system in interaction with a large heat bath will equilibrate and thermalize [16, 17]. For isolated systems it has been shown that "typical" Hamiltonian and observables will be in thermal equilibrium for most times [18, 19, but it seems a very difficult task to decide whether a specific Hamiltonian or observable belongs to this class or not. The thermalization of specific 1D and 2D fermionic and bosonic systems has also been studied 20, 21, 22, 23, 24. Surprisingly, thermalization was not obtained in all the cases. Several reasons have been reported to explain the lack of thermalization in these systems, but it seems that the so-called eigenstate thermalization hypothesis (ETH) plays a fundamental role [23, 24]. The ETH states that thermalization occurs at the level of individual eigenstates [25, 26], whenever they satisfy Berry's conjecture on chaotic eigenfunctions [27], i.e., whenever they behave as (quasi) random superpositions of the basis states. For this and other reasons, the role played in the viability of thermalization by quantum chaos in general, and by the properties of chaotic wave functions in particular, has began to receive some attention [28, 29]. It seems that the number of principal components (NPC) or inverse participation ratio (IPR), which keeps track of the progressive eigenstate delocalization through the integrability to chaos transition, might be directly related to the deviations of the steady expectation values from the corresponding statistical values [30, 31]

In order to get a deeper understanding of the role played by quantum chaos in these processes we study the thermalization of isolated fermionic systems modeled by the simplest two-body random ensemble [32]. Two-body random ensembles are paradigmatic models to study quantum chaos and specially the dynamical transition from integrability to chaos. They have also been used in the past, together with some related models, such as nuclear shell model and interacting spin models, to perform different studies on thermalization. The thermalization criteria were based on the equivalence between different definitions of entropy [33, 34, 35] and temperature [36]; representability of occupancies by Fermi-Dirac distribution [37, 38, 39] (Bose-Einstein distribution for bosons); and calculation of expectation values using the canonical distribution [38]. A brief review of some of these studies can be found in Refs. [40, 41]. However, in the present work, as well as in most recent papers the focus is put on the Ergodicity principle [18, 19] which is the cornerstone for thermalization, and clearly more precise and general that the aforementioned criteria. We study the relevance of several factors in the thermalization process like ETH, the dimension of the Hilbert space, the structure and energy of the initial state and its proximity to the ground state. We also analyze the importance of the degree of chaos as measured by the different chaos markers.

The rest of the paper is organized as follows. Sec. 2 briefly introduces the embedded Gaussian ensembles of random matrices generated by two-body interactions, with emphasis in the embedded Gaussian orthogonal ensemble, and some of the well established main features of the order to chaos transition in this ensemble. However, 
the criteria for determining the transition points as the two-body interaction strength is increased, are somewhat different from those used in the past. Sec. 3 deals with the thermalization of embedded Gaussian orthogonal ensembles. Here we give all the new results of the paper, where the transition points of Sec 2.2 play a central role. After introducing some basic definitions in Sec. 3.1, Sec. 3.2 reports the main numerical results linking thermalization with the type of spectral fluctuations, the delocalization of the wave functions, the structure and the proximity of the initial state to the ground state, or the dimension of the Hilbert space. In Sec. 4 we gather together some analytical results that establish a connection between thermalization, relative to a given observable $\mathcal{O}$, and the value of the NPC for the transition strengths originated at a given eigenstate, or between thermalization and the dimension of the whole Hilbert space, the dimension of the microcanonical energy shell and the correlations generated by $\mathcal{O}$. Finally, Sec. 5 contains the conclusions.

\section{2. $\operatorname{EGOE}(1+2)$ model: order-to-chaos transitions}

\section{1. $E G O E(1+2)$ model}

As previously mentioned, we try to analyze the relationship between the order-tochaos transition and the thermalization of fermionic systems, which can be modeled by an appropriate two-body random matrix ensemble. Here we briefly introduce these ensembles and their relation with quantum chaos. Recent and comprehensive reviews can be found in references [40, 41].

There is a clear relationship between the energy level fluctuation properties of a quantum system and the large time scale behavior of its classical analogue. The spectral fluctuations of a quantum system whose classical analogue is fully integrable are well described by Poisson statistics, i.e., the spacings between successive energy levels are not correlated [42]. According to Bohigas, Gianoni and Schmit [43], the fluctuation properties of generic quantum systems, which in the classical limit are ergodic, coincide with those of the Gaussian ensembles (GE) of random matrices [44]. This statement, initially supported by many experimental data and numerical calculations, has been finally proven for quantum systems with few degrees of freedom, where the semiclassical approximation is valid [45]. The large time scale behavior of the classical analogue also determines the properties of the wave functions. Extensive reviews of later developments can be found in [10, 41] and references there in.

Quantum many-body systems like complex atoms and atomic nuclei are usually considered to be chaotic if their spectral fluctuations are those of the Gaussian orthogonal ensemble (GOE), which is the appropriate GE for systems with time-reversal invariance and rotational symmetry. However, real quantum systems are usually well described by real or effective one- plus two-body interactions in the mean-field basis, whilst GE represent systems with multi-body interactions. The embedded Gaussian ensembles (EGE) of random matrices were introduced to tackle this problem, and to 
provide a more realistic picture of many-body quantum systems. Moreover, in the present context EGE are interesting because random interactions can illustrate the effects on thermalization caused by generic interactions, which lead the system from integrability to chaos.

The EGE $(1+2)$ ensembles consider $m$ fermions or bosons distributed in $n$ singleparticle states $|k\rangle, \quad k=1,2, \cdots, n$, interacting via the following Hamiltonian

$$
H=\sum_{k} \varepsilon_{k} a_{k}^{\dagger} a_{k}+\lambda \sum_{k \leq l, p \leq q}\langle p q|V| k l\rangle a_{p}^{\dagger} a_{q}^{\dagger} a_{l} a_{k},
$$

where the single-particle energies $\varepsilon_{k}$ and the two-body matrix elements (properly symmetrized or antisymmetrized) $\langle p q|V| k l\rangle$ behave as independent Gaussian random variables. In this expression $\lambda$ gives the strength of the two-body interaction, and $a_{k}^{\dagger}$ and $a_{k}$ create and destroy a fermion (or a boson) in the $k$ th single-particle state. In fermionic systems only the strict inequalities $k<l$ and $p<q$ are valid. It has been numerically shown that for $\operatorname{EGE}(2)$ ensembles $\left(\varepsilon_{k}=0\right)$ the spectral fluctuations do agree with those of GE, provided that energy scale is redefined appropriately. Introducing the notation $\langle\bullet\rangle=\langle\langle\bullet\rangle\rangle / d$, where $\langle\langle\bullet\rangle\rangle$ stands for the trace operation, and $d$ is the dimension of the Hilbert space, the centroid and the energy span must be $\langle E\rangle=0$ and $\left\langle E^{2}\right\rangle^{1 / 2}=1$, respectively. [35]

\subsection{The order-to-chaos transition in $\operatorname{EGOE(1+2)}$}

One of the most significant aspects of $\operatorname{EGOE}(1+2)$ is that as $\lambda$ increases, starting from $\lambda=0$, the system undergoes a transition from a regular to a chaotic regime that deeply affects the state density, level fluctuations, and wave functions. This change of dynamical regime is characterized by three chaos markers [40, 41, 46, 47]. There is a first marker $\lambda_{c}$ that signals the transition from Poisson to GOE spectral fluctuations. This transition occurs when the interaction strength $\lambda$ is of the order of the spacing between the basis states that are directly coupled by the residual two-body interaction, a result that came out of nuclear structure calculations by Åberg [48, 49]. More developments in determining the $\lambda_{c}$ marker are given for example in [50, 51]. An important outcome of Aberg criterion is that $\lambda_{c} \propto 1 /\left(m^{2} n\right)$ for $\operatorname{EGOE}(1+2)$, which is well verified in 51. and in the results presented below.

As $\lambda$ increases further from $\lambda_{c}$, the structure of the eigenstates undergoes a deep transformation. First the strength functions change from Breit-Wigner to Gaussian at a transition point denoted by $\lambda_{F}$. Beyond $\lambda_{F}$ we find a third chaos marker $\lambda_{t}$ which defines the center of a region where different definitions of thermodynamic variables, such as entropy, temperature, specific heat, etc., give the same results, as it occurs for infinite systems. As far as the statistical entropy $S^{\text {ther }}$ and the Shanon entropy $S^{\text {inf }}$ are concerned we can understand the meaning of $\lambda_{t}$ as follows. The former is proportional to the logarithm of the state density, which in our case has essentially Gaussian form, though its mean and variance depend on $\lambda$. At $\lambda=0$, where the eigenstates are fully localized in the mean-field basis, information entropy is $S^{i n f}=0$, whilst for sufficiently 
large values of the interaction strength, the eigenstates are quite similar to those of GOE. In our case this occurs for $\lambda \gtrsim 1$ and then $S^{\text {inf }} \approx \log (0.48 d)$, except perhaps near the spectrum edges. Changing from $\lambda=0$ to $\lambda=1$ the wave functions become more and more delocalized in the mean-field basis, being this process faster in the middle than in the spectrum edges, and $\lambda_{t}$ signals the precise value of the residual interaction strength in which the energy dependence of $S^{\text {ther }}$ and $S^{\text {inf }}$ is quite similar. Thus, if we define an appropriate "distance" between the two entropies, it should have a minimum at $\lambda_{t}$. Another property is that $\lambda_{t}$ signals the duality point between $h(1)$ and $V(2)$ basis, i.e., the point where the eigenstates become equally delocalized in the two basis [47, 50]. Beyond $\lambda_{t}$ the eigenstates become quickly similar to those of GOE systems, for which they are essentially Gaussian random superposition of the basis states. This property resembles Berry's conjecture about the ergodic structure of chaotic wave functions in phase space [27]. Nevertheless, a word of caution should be included here. Since $S^{\text {inf }}$ is basis dependent, for $\lambda \gg \lambda_{t}$ it would be more appropriate to define $S^{\text {inf }}$ in the $V(2)$ basis.

For our purposes the markers $\lambda_{c}$ and $\lambda_{t}$ are the most relevant. We detect the change in the spectral fluctuations, and thus the position of $\lambda_{c}$, using the nearestneighbor spacing distribution, denoted $P(s)$. The spacings for generic integrable systems obey the Poisson distribution, i.e, $P(s)=\exp (-s)$, while the Wigner surmise, $P(s)=\frac{\pi}{2} s \exp \left(-\frac{\pi}{4} s^{2}\right)$, provides a very good approximation for GOE-like systems [44]. The Brody distribution [35],

$$
P(s, \omega)=A_{\omega}(\omega+1) s^{\omega} \exp \left(-A_{\omega} s^{\omega+1}\right),
$$

where $\omega$ is usually called the Brody parameter and $A_{\omega}$ is a normalization constant, it is used to asses how close the fluctuations are to the Poisson limit, corresponding to $\omega=0$, or to the Wigner surmise with $\omega=1$ [52]. Although Eq. (21) is only a heuristic formula, and the Brody parameter has no definite meaning for Hamiltonian systems, it has been employed in a variety of studies since its introduction. Very recently a physical foundation of $\omega$ has been found by Sakhr and Nieminen in the context of self-similar fractals [53]. As the interaction strength in Eq. (11) increases from $\lambda=0$ to a sufficiently large value, the Brody parameter changes from $\omega=0$ to $\omega \simeq 1$. In what follows, the position of the first chaos marker $\lambda_{c}$ is fixed by the condition $\omega(\lambda)=1 / 2$. Similar conditions can be found in the literature [51, 54].

To locate the value of the third marker $\lambda_{t}$ we consider the values of three different entropies:

- Thermodynamic entropy, $S_{E}^{\text {ther }}=\log \rho(E)$, where $\rho(E)$ is the density of states.

- Information entropy in the mean-field basis $S_{E}^{\text {inf }}=-\sum_{k=1}^{d}\left|c_{k}^{E}\right|^{2} \log \left|c_{k}^{E}\right|^{2}$, where the coefficients $c_{k}^{E}$ are the eigenstates components in the mean-field basis.

- Single-particle entropy $S_{E}^{s p}=-\sum\left\{\left\langle n_{i}\right\rangle^{E} \log \left(\left\langle n_{i}\right\rangle^{E}\right)+\left(1-\left\langle n_{i}\right\rangle^{E}\right) \log \left(1-\left\langle n_{i}\right\rangle^{E}\right)\right\}$, with $\left\langle n_{i}\right\rangle^{E}$ the occupancy of the $i$ th single-particle state at energy $E$. 
Entropies formulas, valid for $\lambda>\lambda_{F}$, are given in [34, 55] for $\operatorname{EGOE}(1+2)$ ensembles. If we define the average "distance" between the three entropies as

$$
\Delta_{s}(\lambda)=\left\{\int_{-\infty}^{\infty}\left[\left(R_{E}^{\text {inf }}-R_{E}^{\text {ther }}\right)^{2}+\left(R_{E}^{s p}-R_{E}^{\text {ther }}\right)^{2}\right] d E\right\}^{1 / 2} / \int_{-\infty}^{\infty} R_{E}^{\text {ther }} d E,
$$

where $R_{E}^{\alpha}=\exp \left\{S_{E}^{\alpha}-S_{\max }^{\alpha}\right\}$, the value of $\lambda_{t}$ corresponds to the minimum of $\Delta_{s}$ because this ensures that the values of the different entropies will be very close to each other.

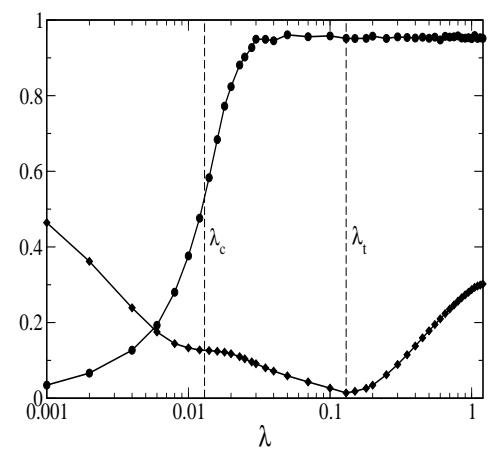

Figure 1. Average values $\overline{\omega(\lambda)}$ (dots) and $\overline{\Delta_{s}(\lambda)}$ (diamonds) for $\lambda \in[0,1]$, calculated using 60 members of a $\operatorname{EGOE}(1+2)$ with $m=6$ and $n=16$. The vertical dashed lines indicate the position of $\lambda_{c}$ and $\lambda_{t}$.

Fig. 1 displays the ensemble averages $\overline{\omega(\lambda)}$ and $\overline{\Delta_{s}(\lambda)}$ along $\lambda \in[0,1]$, for a 60 member $\operatorname{EGOE}(1+2)$ with $m=6$ and $n=16$. In order to enlarge the region where the order-to-chaos transition takes place we use a logarithmic horizontal axis. The two vertical dashed lines indicate the respective positions of $\lambda_{c}$ and $\lambda_{t}$. It can be seen that $\lambda_{c} \simeq 0.013$ and $\lambda_{t} \simeq 0.13$, values that are consistent with theoretical estimates (see [40, 41, 46]). For $\lambda \simeq 0.03$ we obtain $\omega \simeq 0.96$, which is very close to the actual GOE result. However, the structure of these states is still very different to those of a GOE system because $\overline{S^{i n f}} \simeq \overline{S_{G O E}^{i n f}} / 2$. Only when $\lambda \simeq 1$ one finds that $\overline{S^{i n f}} \simeq \overline{S_{G O E}^{\text {inf }}}$, signaling that the dynamics has become fully chaotic.

\section{Thermalization definitions and numerical results}

\subsection{Basic definitions}

We study the thermalization properties of finite fermionic systems with time-reversal and rotational invariance. To be precise we consider $m$ fermions distributed in $n$ independent particle states, interacting via the Hamiltonian (1), where $\varepsilon_{k}$ and $\langle p q|V| k l\rangle$ are independent real Gaussian random variables. These systems are usually called $\operatorname{EGOE}(1+2)$ ensembles. In this work we use $\overline{\varepsilon_{k}}=k, \overline{\left(\epsilon_{k}-k\right)^{2}}=1 / 2, \overline{\langle p q|V| k l\rangle}=0$, $\overline{|\langle p q|V| k l\rangle|^{2}}=1+\delta_{(p q),(k l)}$, and the energy scale is such that $\langle E\rangle=0$ and $\left\langle E^{2}\right\rangle^{1 / 2}=1$, regardless of the value of the interaction strength $\lambda$. The results presented below have 
Thermalization in the Two-Body Random Ensemble

\begin{tabular}{c|cccccccccc}
\hline$m$ & 5 & 5 & 5 & 5 & 5 & 6 & 6 & 6 & 6 & 6 \\
$n$ & 12 & 13 & 14 & 15 & 16 & 12 & 13 & 14 & 15 & 16 \\
$d$ & 792 & 1287 & 2002 & 3003 & 5005 & 924 & 1716 & 3003 & 5005 & 8008 \\
\hline
\end{tabular}

Table 1. Matrix dimension for EGOE systems with $m=5,6$ and $n=12-16$

been obtained by fully diagonalizing 60 member $\operatorname{EGOE}(1+2)$ systems with $m=5,6$, $n=12-16$ and $\lambda \in[0,1]$. The corresponding dimensions are given in Table 1,

Let $|\Psi(0)\rangle$ be the initial state of the system, that we decompose as

$$
|\Psi(0)\rangle=\sum_{\mu} C_{\mu}\left|E_{\mu}\right\rangle
$$

in the eigenstate basis $\left\{\left|E_{\mu}\right\rangle, \mu=1,2, \ldots d\right\}$ of the Hamiltonian (1). Then, given a certain observable $\mathcal{O}$ we define:

- The instantaneous value $O(t)=\langle\Psi(t)|\mathcal{O}| \Psi(t)\rangle=\left\langle\left\langle\mathcal{O} \rho_{\Psi}(t)\right\rangle\right\rangle$, where $\rho_{\Psi}(t)=$ $|\Psi(t)\rangle\langle\Psi(t)|$.

- The time average $\langle O(t)\rangle_{T}=(2 T)^{-1} \int_{t-T}^{t+T} O(\tau) \mathrm{d} \tau$. When $T \gg 1,\langle O(t)\rangle_{T}{ }^{T \gg 1}$ $\langle\mathcal{O}\rangle_{e q}=\sum_{\mu}\left|C_{\mu}\right|^{2} D_{o}\left(E_{\mu}\right)$, where $D_{o}\left(E_{\mu}\right)=\left\langle E_{\mu}|\mathcal{O}| E_{\mu}\right\rangle$. The steady state average can also be written as $\langle\mathcal{O}\rangle_{e q}=\left\langle\left\langle\mathcal{O} \rho_{e q}\right\rangle\right\rangle$, with $\rho_{e q}=\sum_{\mu}\left|C_{\mu}\right|^{2}\left|E_{\mu}\right\rangle\left\langle E_{\mu}\right|$.

- The statistical average $\langle\mathcal{O}\rangle_{\text {stat }}=\left\langle\left\langle\mathcal{O} \rho_{\text {stat }}\right\rangle\right\rangle$, where $\rho_{\text {stat }}$ is the density operator corresponding to an appropriate statistical ensemble.

We say that the system thermalizes if for any relevant observable $\mathcal{O}$ and almost any state $|\Psi(t)\rangle$, it is satisfied that

$$
\langle O(t)\rangle_{T}{ }^{T \gg 1}\langle\mathcal{O}\rangle_{e q} \approx\langle\mathcal{O}\rangle_{\text {stat }}
$$

In practice, to asses whether Eq. (5) do approximately holds for a specific observable, one can use the relative error

$$
\Delta_{o}=\left|\frac{\delta_{o}}{\langle\mathcal{O}\rangle_{\text {stat }}}\right|=\left|\frac{\langle\mathcal{O}\rangle_{\text {eq }}-\langle\mathcal{O}\rangle_{\text {stat }}}{\langle\mathcal{O}\rangle_{\text {stat }}}\right|
$$

which is well suited to compare the degree of thermalization of different systems, or the thermalization of a single system relative to distinct observables.

Let us consider that the system is prepared in a non-equilibrium state $|\Psi(0)\rangle$ for which the energy is essentially constant. Hamiltonian eigenvectors are excluded as they are stationary states, but we can assume that $\langle\Psi(0)|H| \Psi(0)\rangle=E_{0}$, and $\left[\left\langle\Psi(0)\left|H^{2}-E_{0}^{2}\right| \Psi(0)\right\rangle\right]^{1 / 2}<\Delta E$, with $\Delta E$ sufficiently small compared to the energy spectrum span, but large enough to contain many energy eigenstates. In such a case the microcanonical ensemble is the preferred statistical ensemble. Denoting by $W$ the corresponding energy shell, i.e., $W=\left\{\left|E_{\mu}\right\rangle ; E_{\mu} \in\left[E_{0}-\Delta E, E_{0}+\Delta E\right]\right\}$, the microcanonical density operator is 


$$
\rho_{m c}=\frac{1}{d^{\prime}} \sum_{\mu}^{\prime}\left|E_{\mu}\right\rangle\left\langle E_{\mu}\right|,
$$

where $d^{\prime}$ is the dimension of the subspace $W$ and the symbol $\sum^{\prime}$ means that the sum is restricted to eigenstates belonging to $W$. Thus, the corresponding microcanonical average is given by

$$
\langle\mathcal{O}\rangle_{m c}=\frac{1}{d^{\prime}} \sum_{\mu}^{\prime} D_{o}\left(E_{\mu}\right) .
$$

To examine whether the energy plays a role in the thermalization of the system we shall use three different energy intervals with $\Delta_{E}=0.1$ and $E_{0}=0.0,1.0$ and 1.7. We denote the corresponding energy shells by $W_{1}, W_{2}$ and $W_{3}$ respectively. Moreover, to see how the initial conditions affect the process we let the system evolve from three different types of initial states, defined as:

- $\left|\Psi^{(1)}(0)\right\rangle \propto P_{W}\left|k_{0}\right\rangle$, where $P_{W}$ is the projector onto $W$, and $\left|k_{0}\right\rangle$ is the mean-field state with energy $e=E_{0}$.

- $\left|\Psi^{(2)}(0)\right\rangle \propto \sum_{\mu}^{\prime} C_{\mu}\left|E_{\mu}\right\rangle$, with the coefficients $C_{\mu}$ Gaussian random variables with mean zero and variance equal to one, i.e., $C_{\mu}=\mathcal{G}(0,1)$.

- $\left|\Psi^{(3)}(0)\right\rangle \propto \sum_{\mu}^{\prime} C_{\mu}\left|E_{\mu}\right\rangle$, where the expansion coefficients are $C_{\mu}=$ $e^{-\alpha\left(\frac{E_{\mu}-E_{0}}{\Delta E}\right)^{2}} \mathcal{G}(0,1)$.

The states $\Psi^{(2)}(0)$ and $\Psi^{(3)}(0)$ are random superpositions of the eigenstates belonging to $W$, but due to the Gaussian factor the distribution of the $C_{\mu}$ coefficients is wider for $\Psi^{(3)}(0)$. As we shall see below the distribution of the state amplitudes inside $W$ is one of the factors that may affect the thermalization process.

Before we turn to the main results, let us introduce the four types of generic observables for which we study the validity of Eq. (5):

- diagonal one-body operators $\mathcal{O}_{d}(1)=\sum_{k} \theta_{k} a_{k}^{\dagger} a_{k}$,

- one-body operators $\mathcal{O}(1)=\sum_{k, l} \theta_{k l} a_{k}^{\dagger} a_{l}$,

- two-body operators $\mathcal{O}(2)=\sum_{k<l, p<q} \theta_{k l p q} a_{k}^{\dagger} a_{l}^{\dagger} a_{q} a_{p}$,

- strength function operators $\mathcal{O}_{s f}=\mathcal{O}^{T}(1) \mathcal{O}(1)$,

where the parameters $\theta_{k}, \theta_{k l}$ and $\theta_{k l p q}$ are taken as random variables.

\subsection{Numerical results}

Let us consider the ensemble averages $\overline{\Delta_{o_{d}(1)}}, \overline{\Delta_{o(1)}}, \overline{\Delta_{o(2)}}$, and $\overline{\Delta_{o_{s f}}}$ for a 60 member EGOE with $(m, n)=(6,16)$. Fig. 2 displays their evolution with the strength $\lambda$ when the system is prepared at $t=0$ in a state $\Psi^{(1)}(0)$ with energy $E \simeq E_{0}=0$ ( $\left.\Psi^{(1)}(0) \in W_{1}\right)$. Because of the large differences between the relative errors of these operators and to properly visualize their evolution in the short interval $\lambda_{c} \leq \lambda \leq \lambda_{t}$, 


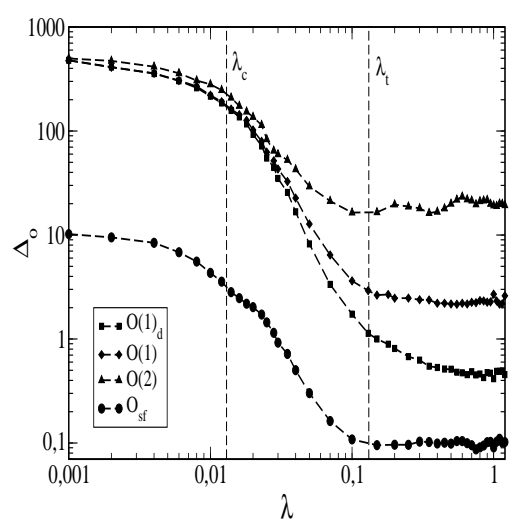

Figure 2. Evolution with the interaction strength $\lambda$ of the averages $\overline{\Delta_{o_{d}(1)}}$ (squares), $\overline{\Delta_{o(1)}}$ (diamonds), $\overline{\Delta_{o(2)}}$ (triangles), and $\overline{\Delta_{o_{s f}}}$ (dots), given in percent, for a 60 member $\operatorname{EGOE}(1+2)$ with $(m, n)=(6,16)$ initially prepared in a state $\Psi^{(1)}(0) \in W_{1}$.

a $\log$ - $\log$ scale is used. As for Fig. 1 the two vertical lines give the positions of $\lambda_{c}$ and $\lambda_{t}$. In all the cases $\overline{\Delta_{o}}$ becomes smaller as the interaction strength increases up to $\lambda \approx \lambda_{t}$. It is very important to realize that the transition from Poisson to GOE spectral fluctuations, which is considered the most relevant signature of quantum chaos, occurs at $\lambda \approx \lambda_{c}$ and does not modify this trend. On the contrary, for $\lambda>\lambda_{t}$ the relative errors either remain essentially constant or the decreasing rate is much smaller. Recall that $\lambda_{t}$ defines a region where the three entropies $S_{E}^{\text {ther }}, S_{E}^{\text {inf }}$ and $S_{E}^{s p}$ take essentially the same values, and signals the point at which the wave functions start to become very delocalized in the mean-field basis. Beyond $\lambda_{t}, \overline{\Delta_{O}}$ becomes clearly smaller than one percent only for two operators, namely $\mathcal{O}_{d}(1)$ and $\mathcal{O}_{s f}$. Their errors are $\overline{\Delta_{o_{d}(1)}} \approx 0.5 \%$ and $\overline{\Delta_{o_{s f}}} \approx 0.1 \%$, respectively. Thus, as long as the the system is prepared in an initial state $\Psi^{(1)}(0) \in W_{1}$ and $\lambda>\lambda_{t}$, Eq. (5) approximately holds for the observables $\mathcal{O}_{d}(1)$ and $\mathcal{O}_{s f}$ and then we can assert that the system thermalizes relative to these observables. This is not the case of the observables $\mathcal{O}(1)$ and $\mathcal{O}(2)$. It is worth noting that perhaps the main difference between $\mathcal{O}(1), \mathcal{O}(2)$ in one hand, and $\mathcal{O}_{d}(1), \mathcal{O}_{s f}$ in the other, is that the latter have meaningful smoothed form for large $\lambda$, as given by spectral distribution methods [41, 56, 57].

The results corresponding to other choices of $\Psi(0) \in W_{1}$ are shown in Fig. 3. For simplicity we have represented only the results for $\mathcal{O}_{s f}$ and $\mathcal{O}(1)$. Curves in black, red and green correspond to $\Psi^{(1)}(0), \Psi^{(2)}(0)$ and $\Psi^{(3)}(0)$, respectively. In all cases the initial state belongs to the energy shell $W_{1}$. We see that the choice of the initial conditions does not affect the main trend: $\overline{\Delta_{o(1)}}$, and $\overline{\Delta_{o_{s f}}}$ diminish progressively as the strength $\lambda$ is increased, and once $\lambda>\lambda_{t}$ their values remain essentially constant. However, the precise values are quite different. When $\lambda>\lambda_{t}$, the initial states $\Psi^{(1)}(0)$ and $\Psi^{(2)}(0)$ give rise to very similar results while the error corresponding to $\Psi^{(3)}(0)$ is clearly larger.

To shed some light on the mechanisms leading to these results lets us note that the 


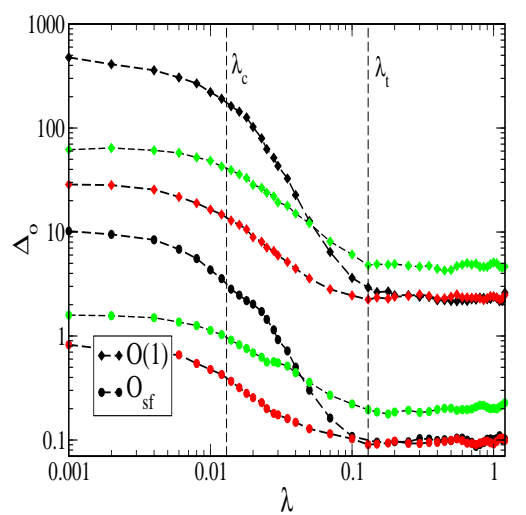

Figure 3. Values of $\overline{\Delta_{o(1)}}$ (diamonds) and $\overline{\Delta_{o_{s f}}}($ dots), expressed in percent, as function of $\lambda$ in a 60 member $\operatorname{EGOE}(1+2)$ with $(m, n)=(6,16)$, and initial conditions given by $\Psi^{(1)}(0)$ (black), $\Psi^{(2)}(0)$ (red), and $\Psi^{(3)}(0)$ (green). In all cases the initial state belongs to the energy shell $W_{1}$.

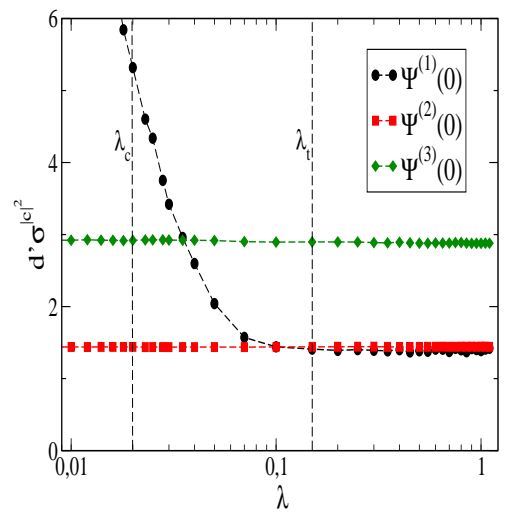

Figure 4. Variation with the interaction strength $\lambda$ of the standard deviation $\sigma_{\left|C_{\mu}\right|^{2}}$ of the initial state $\Psi(0)$ components in the energy eigenbasis. The values of $\sigma_{\left|C_{\mu}\right|^{2}}$ have been multiplied by the dimension $d^{\prime}$ of the energy shell. The results for $\Psi^{(1)}(0) \in W_{1}$ (black), $\Psi^{(2)}(0) \in W_{1}\left(\right.$ red), and $\Psi^{(3)}(0) \in W_{1}$ (green) are plotted

difference

$$
\delta_{0}=\langle\mathcal{O}\rangle_{e q}-\langle\mathcal{O}\rangle_{m c}=\sum_{\mu}^{\prime}\left(\left|C_{\mu}\right|^{2}-\frac{1}{d^{\prime}}\right) D_{o}\left(E_{\mu}\right),
$$

becomes very small when: the distribution of the coefficients $C_{\mu}$ is nearly flat, i.e, $\left|C_{\mu}\right|^{2} \approx 1 / d^{\prime}$, or the matrix elements $D_{o}\left(E_{\mu}\right)$ almost do not fluctuate inside the energy shell $W$, and therefore $D_{o}\left(E_{\mu}\right) \approx\langle\mathcal{O}\rangle_{m c}$. This condition is known in the literature as the eigenstate thermalization hypothesis (ETH), which has been conjectured to hold in chaotic quantum systems [22, 26]. The standard deviation of the initial state components in the energy eigenbasis, $\sigma_{\left|C_{\mu}\right|^{2}}$, is plotted in Fig. 4. When $\lambda$ is very small $\sigma_{\left|C_{\mu}\right|^{2}}$ takes very different values in the three cases, being quite larger for $\Psi^{(1)}(0)$. However, in this 
case $\sigma_{\left|C_{\mu}\right|^{2}}$ undergoes a sharp decreasing up to $\lambda \simeq \lambda_{t}$, where its values are very similar to those of $\Psi^{(2)}(0)$ and half those of $\Psi^{(3)}(0)$.

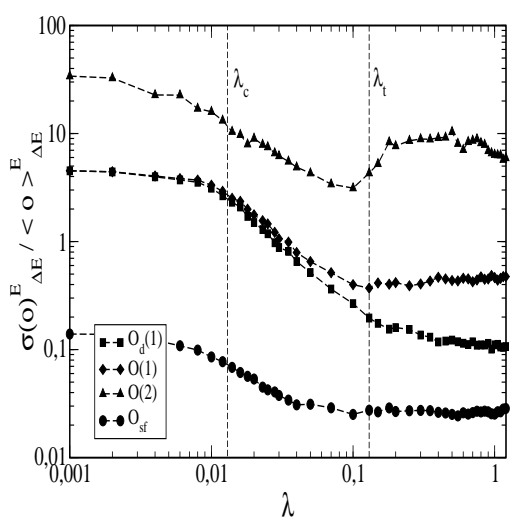

Figure 5. Evolution with $\lambda$ of the matrix elements $\left\langle E_{\mu}|\mathcal{O}| E_{\mu}\right\rangle$ fluctuations in the central energy shell $W_{1}$, measured by the ratio $\kappa_{o}=\sigma_{o}^{m c} /\langle\mathcal{O}\rangle_{m c}$ (see text). Results for $\mathcal{O}_{d}(1)$ (squares), $\mathcal{O}(1)$ (diamonds), $\mathcal{O}(2)$ (triangles) and $\mathcal{O}_{s f}$ (circles).

Defining the standard deviation of the expectation values $D_{o}\left(E_{\mu}\right)$ inside an energy shell $W$ as

$$
\sigma_{o}^{m c}=\left[\frac{1}{d^{\prime}} \sum_{\mu}^{\prime}\left\langle E_{\mu}\left|\mathcal{O}-\langle\mathcal{O}\rangle_{m c}\right| E_{\mu}\right\rangle^{2}\right]^{1 / 2},
$$

the ratio $\kappa_{o}=\sigma_{o}^{m c} /\langle\mathcal{O}\rangle_{m c}$ provides us a measure of the fluctuations of these matrix elements. Fig. 5 plots $\kappa_{o}(\lambda)$ for a randomly selected member of the ensemble inside $W_{1}$. Throughout the interval $\lambda=0$ to $\lambda=1$ the ratio $\kappa_{o}$ is reduced sharply by a factor ranging between 5 and 40 , but even then we get $\kappa_{o} \gtrsim 1$ for operators like $\mathcal{O}(1)$ and $\mathcal{O}(2)$. Remarkably, $\kappa_{o} \lesssim 0.1$ for $\mathcal{O}_{d}(1)$ or $\mathcal{O}_{s f}$, meaning that the fluctuations of their expectation values are small compared to $\langle\mathcal{O}\rangle_{m c}$ in the energy shell $W_{1}$.

The extraordinary resemblance of Fig. 5 with Figs. 2 and 3 shows that the fluctuations of the eigenstate expectation values determine for which observables thermalization occurs. In other words, the thermalization of the system is correlated with the degree of compliance of the ETH. Moreover, given a certain observable the exact degree of thermalization depends on the width of the initial state: the wider is the initial state the worse thermalizes the system, a fact that is in agreement with some previous claims [58].

Thermalization may be affected by other factors, such as the proximity of $E_{0}$ to the edges of the spectrum, and in particular to the ground-state energy. In order to explore this question we have compared the behavior of $\Delta_{o}$ in three energy shells $W_{1}$, $W_{2}$ and $W_{3}$, of width $\Delta_{E}=0.1$ and $E_{0}=0.0,1.0$ and 1.7, respectively. Although the energies of the largest eigenstates oscillate between 2.5 and 3 depending on the value of $\lambda$, it is very difficult to get closer to the spectrum end. The reason is that outside the 


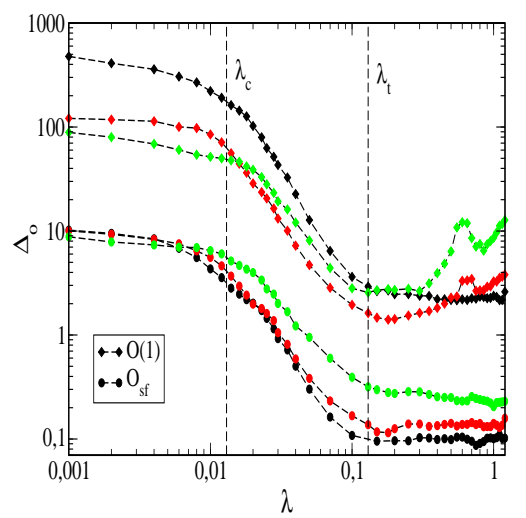

Figure 6. Behavior of the ensemble averages $\overline{\Delta_{o(1)}}$ (diamonds) and $\overline{\Delta_{o_{s f}}}$ (dots) as $\lambda$ varies, for three different initial state energies, $E_{0}=0$ (black), $E_{0}=1$ (red), and $E_{0}=1.7$ (green).

central interval $(-2,2)$ the state density is so scarce that it is impossible to obtain a narrow energy shell which contains a large number of states $d^{\prime}$, but small compared to the dimension $d$ of the whole space. We plot in Fig. 66 the variation with $\lambda$ of the two averages $\overline{\Delta_{o_{s f}}}$ and $\overline{\Delta_{o(1)}}$, calculated for an EGOE system with $(m, n)=(6,16)$, which is initially prepared in a state of type $\Psi^{(1)}(0)$. One sees immediately that $\overline{\Delta_{o}}$ behaves very differently for these operators. Once the thermalization region $\left(\lambda>\lambda_{t}\right)$ has been reached, the relative error $\overline{\Delta_{o_{s f}}}$ grows as the energy $E_{0}$ approaches the end of the energy spectrum. By contrast, the evolution of $\overline{\Delta_{o(1)}}$ is more irregular, but still this error is clearly larger for $E_{0} \approx 1.7$ and $\lambda \approx 1$. Therefore, the proximity of the initial state energy to the spectrum edges inhibits the thermalization of the system.

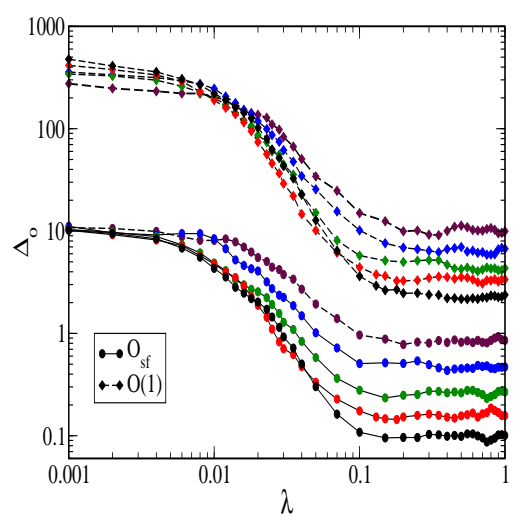

Figure 7. Variation with $\lambda$ of $\overline{\Delta_{o(1)}}$ (diamonds), and $\overline{\Delta_{o_{s f}}}$ (dots) in five ensembles with dimensions $d=924$ (maroon), 1716 (blue), 3003 (green), 5005 (red), 8008 (black), and initial conditions given by $\Psi^{(1)}(0) \in W_{1}$.

Before closing this section we shall briefly consider whether the dimension of the 
$(m, n)$ Hilbert space hinders or enhances thermalization. For illustration we consider 5 systems with $(m, n)=(6,12),(6,13),(6,14),(6,15)$ and $(6,16)$, with dimensions (see Table 1) $d=924,1716,3003,5005$ and 8008 , respectively. Fig. 7 shows the evolution of $\overline{\Delta_{o}}$ for $\mathcal{O}_{s f}$ and $\mathcal{O}(1)$ as the dynamical regime changes from regularity at $\lambda=0$ to full chaos for $\lambda \simeq 1$. The growth rate of $d$ is very small, but it is enough to induce a gentle decrease in $\overline{\Delta_{o}}$, while maintaining the same qualitative trend. Similar results are obtained for the other two observables $\mathcal{O}_{d}(1)$ and $\mathcal{O}(2)$. If we also take into account the analytical results of the next section we can conclude that the system will always thermalize in the thermodynamic limit (the difference between microcanonical and diagonal predictions decreases with system size), but there may be relevant differences for finite dimensions, which is important since many physical systems of interest are mesoscopic.

\section{Analytical results for thermalization}

Our goal now is to relate the relative error $\Delta_{o}$ with the fluctuation properties of the eigenstates and with the correlations generated by the observable $\mathcal{O}$. To this end let us focus on the quantity

$$
\delta_{0}=\langle\mathcal{O}\rangle_{e q}-\langle\mathcal{O}\rangle_{m c}=\left\langle\left\langle\mathcal{O}\left(\rho_{e q}-\rho_{m c}\right)\right\rangle\right\rangle=\langle\langle\mathcal{O} \Delta \rho\rangle\rangle,
$$

with $\Delta \rho=\rho_{e q}-\rho_{m c}$. It can be considered as a random variable because we model the physical system by means of an appropriate ensemble and thus the external products $\left|E_{\mu}\right\rangle\left\langle E_{\mu}\right|$ appearing in $\Delta \rho$ change from one member of the Hamiltonian ensemble to another. Moreover, being interested in "typical" properties we can introduce a fictitious ensemble of initial states, $|\Psi(0)\rangle=\sum_{\mu}^{\prime} C_{\mu}\left|E_{\mu}\right\rangle$, with similar energies. Assuming that they are uniformly distributed in the unit sphere in $W$, the fluctuation properties of the $C_{\mu}$ coefficients obey the Porter-Thomas (P-T) distribution [35] whenever $d^{\prime}$ is large enough. Thus

$$
\overbrace{\rho_{e q}}=\sum_{\mu}^{\prime} \overbrace{\left|C_{\mu}\right|^{2}}\left|E_{\mu}\right\rangle\left\langle E_{\mu}\left|=\frac{1}{d^{\prime}} \sum_{\mu}^{\prime}\right| E_{\mu}\right\rangle\left\langle E_{\mu}\right|=\rho_{m c},
$$

where $\overbrace{}^{-}$means averaging over the fictitious ensemble of initial states. Therefore $\overbrace{\Delta \rho}=0$ implying that $\overbrace{\delta_{0}}^{2}=\overbrace{\delta_{0}}=0$. Then, $\overbrace{\delta_{0}^{2}}$ is the variance of (9) and we may agree on defining the "typical" value of $\Delta_{o}$ as

$$
\Delta_{o}^{t y p}=\left[\frac{\widetilde{\delta_{0}^{2}}}{\overline{\langle\mathcal{O}\rangle_{m c}^{2}}}\right]^{1 / 2}
$$

Introducing the shifted operator $\mathcal{Q}=\mathcal{O}-a I$, with $a \in \mathbb{R}$, it is easy to see that $\delta_{0}=\delta_{Q}$. Moreover, a straightforward calculation gives

$$
\overbrace{\delta_{0}^{2}}=\sum_{\mu, \nu}^{\prime} \overbrace{D_{Q}\left(E_{\mu}\right) D_{Q}\left(E_{\nu}\right)} \overbrace{\left(\left|C_{\mu}\right|^{2}-\frac{1}{d^{\prime}}\right)\left(\left|C_{\nu}\right|^{2}-\frac{1}{d^{\prime}}\right)},
$$


and taking into account the fluctuation properties of the $C_{\mu}$ coefficients we obtain

$$
\overbrace{\delta_{0}^{2}}=\frac{2}{d^{\prime 2}} \sum_{\mu} \overline{D_{Q}^{2}\left(E_{\mu}\right)} .
$$

Introducing this result in (11), the value of $\Delta_{o}^{\text {typ }}$ is given by the expression

$$
\Delta_{o}^{t y p}=\left[2 \frac{\sum_{\mu}^{\prime} \overline{D_{Q}^{2}\left(E_{\mu}\right)}}{\left(\sum_{\mu}^{\prime} \overline{D_{o}\left(E_{\mu}\right)}\right)^{2}}\right]^{1 / 2}
$$

which can be further simplified because the averaged forms $\overline{D_{Q}^{n}\left(E_{\mu}\right)}$ are smooth functions of the energy that do not change substantially inside the energy window $W$. Thus, we arrive to

$$
\Delta_{o}^{t y p}=\left[\frac{2}{d^{\prime}} \frac{\overline{D_{Q}^{2}\left(E_{0}\right)}}{\left(\overline{D_{o}\left(E_{0}\right)}\right)^{2}}\right]^{1 / 2}
$$

Since $a$ is a free parameter we can choose $a=\overline{D_{o}\left(E_{0}\right)}$, which gives rise to

$$
\Delta_{o}^{t y p}=\left[\frac{2}{d^{\prime}} \frac{\overline{D_{o}^{2}\left(E_{0}\right)}-\left(\overline{D_{o}\left(E_{0}\right)}\right)^{2}}{\left(\overline{D_{o}\left(E_{0}\right)}\right)^{2}}\right]^{1 / 2}=\left[\frac{2}{d^{\prime}} \frac{\overline{\sigma_{o}^{2}\left(E_{0}\right)}}{\left(\overline{D_{o}\left(E_{0}\right)}\right)^{2}}\right]^{1 / 2}
$$

For strength function operators, like $\mathcal{O}_{s f}=\mathcal{O}^{T} \mathcal{O}$, the value of $\Delta_{o}^{\text {typ }}$ can be related to the NPC (also called IPR) in transition strengths originating from the central eigenstate $\left|E_{0}\right\rangle$. Before we proceed it seems suitable to define the usual NPC for eigenstates and its extension for transition strengths. The former is a measure of the eigenstate complexity in the mean-field basis of Slater determinants $|K\rangle$ with $K \equiv k_{1}, k_{2}, \ldots, k_{m}$ and $k_{i}=1,2, \ldots, n$. Expanding the eigenstates in this basis, i.e.,

$$
\left|E_{\mu}\right\rangle=\sum_{K=1}^{d} U_{K}\left(E_{\mu}\right)|K\rangle,
$$

where the amplitudes $U_{K}\left(E_{\mu}\right)$ satisfy that $\sum_{K=1}^{d}\left|U_{K}\left(E_{\mu}\right)\right|^{2}=1$, the number of principal components is defined as

$$
\operatorname{NPC}(E)=\left[\sum_{K}\left|U_{K}\left(E_{\mu}\right)\right|^{4}\right]^{-1} .
$$

Note that NPC, as a function of the amplitudes $U$, attains its absolute minimum $\mathrm{NPC}_{\text {min }}=1$ whenever the wave function is localized in a single basis state, and its absolute maximum $\mathrm{NPC}_{\max }=d$ when the wave function is completely delocalized and all the amplitudes are equal to $\left|U_{K}\left(E_{\mu}\right)\right|^{2}=1 / d$. Generally speaking NPC gives the effective number of basis states that build up Hamiltonian eigenstates. Thus, it is small 
for localized states, while for chaotic states, which are quite delocalized in the meanfield basis, it takes values comparable but somewhat smaller than $d$ because system symmetries and orthogonality prevent reaching its maximum. For instance, the average value for $\mathrm{GOE}$ is $\overline{\mathrm{NPC}}^{\mathrm{GOE}}=d / 3$.

Since the analysis of eigenvector amplitudes is largely equivalent to dealing with transition strengths we can extend the previous discussion. Using the standard notation of spectral distribution methods, let us introduce the so called locally normalized strength $\hat{R}$, and the normalized stregth $\mathcal{R}$ generated by the action of the operator $\mathcal{O}$ on a certain eigenstate $\left|E_{\mu}\right\rangle$ as

$$
\begin{aligned}
& \hat{R}\left(E_{\nu}, E_{\mu}\right)=\left[\overline{\left|\left\langle E_{\nu}|\mathcal{O}| E_{\mu}\right\rangle\right|^{2}}\right]^{-1}\left|\left\langle E_{\nu}|\mathcal{O}| E_{\mu}\right\rangle\right|^{2}, \\
& \mathcal{R}\left(E_{\nu}, E_{\mu}\right)=\left[M_{0}\left(E_{\mu}\right)\right]^{-1}\left|\left\langle E_{\nu}|\mathcal{O}| E_{\mu}\right\rangle\right|^{2},
\end{aligned}
$$

where $M_{0}\left(E_{\mu}\right)=\left\langle E_{\mu}\left|\mathcal{O}^{T} \mathcal{O}\right| E_{\mu}\right\rangle$ is the total strength sum. In our notation $M_{0}\left(E_{\mu}\right)=$ $D_{O_{s f}}\left(E_{E_{\mu}}\right)$. Note that the initial and final spaces connected by $\mathcal{O}$ do not need to be the same, but for simplicity we shall consider here a single space. If we normalize the state vector $\mathcal{O}\left|E_{\mu}\right\rangle$ and expand it in the Hamiltonian eigenbasis

$$
\left[M_{0}\left(E_{\mu}\right)\right]^{-1} \mathcal{O}\left|E_{\mu}\right\rangle=\sum_{\nu=1}^{d} C_{\nu}\left|E_{\nu}\right\rangle=\sum_{\nu=1}^{d}\left[M_{0}\left(E_{\mu}\right)\right]^{-1}\left\langle E_{\nu}|\mathcal{O}| E_{\mu}\right\rangle\left|E_{\nu}\right\rangle,
$$

then the NPC for the strength distribution generated by $\mathcal{O}$ on the eigenstate $\left|E_{\mu}\right\rangle$ is defined as

$$
\operatorname{NPC}\left(E_{\mu}\right)=\left[\sum_{\nu}\left|C_{\nu}\right|^{4}\right]^{-1}=\left[\sum_{\nu}\left(\mathcal{R}\left(E_{\nu}, E_{\mu}\right)\right)^{2}\right]^{-1} .
$$

Contrary to usual NPC in wavefunctions it only depends on the operator and the initial state, but it is basis independent. The NPC for strength distributions gives the effective number of eigenstates over which the strength generated by the action of the operator $\mathcal{O}$ on a given eigenstate is spread. For operators generating collective states (then $H$ and $\mathcal{O}$ are highly correlated) NPC should be small, while for operators generating chaotic states it should take large values.

Before turning back to the calculation of $\Delta_{o_{s f}}^{t y p}$ we give an expression for the smoothed $\mathrm{NPC}_{E_{\mu}}$ that will be used below. If we assume that the fluctuations in the locally renormalized strengths $\hat{R}\left(E_{\nu}, E_{\mu}\right)$ follow the P-T distribution then $\overline{\hat{R}}=1$, $\overline{\hat{R}^{2}}=3$, and

$$
\begin{aligned}
\overline{\mathrm{NPC}}_{E_{\mu}}^{\mathrm{EGOE}} & =\overline{\left[\sum_{\nu}\left(\mathcal{R}\left(E_{\nu}, E_{\mu}\right)\right)^{2}\right]^{-1}}=\left[3 \sum_{\nu}\left(\overline{\mathcal{R}\left(E_{\nu}, E_{\mu}\right)}\right)^{2}\right]^{-1} \\
& =\left[3 \sum_{\nu} \frac{\left(\overline{\left\langle E_{\nu}|\mathcal{O}| E_{0}\right\rangle^{2}}\right)^{2}}{\left(\overline{M_{0}\left(E_{0}\right)}\right)^{2}}\right]^{-1}
\end{aligned}
$$


An $\operatorname{EGOE}(1+2)$ formula for NPC in transitions strengths and $\lambda$ not too far from $\lambda_{t}$ is given in Ref. [61. The typical error $\Delta_{o_{s f}}^{t y p}$ can be written as

$$
\Delta_{o_{s f}}^{t y p}=\left[\frac{2}{d^{\prime}} \frac{\overline{D_{o_{s f}}^{2}\left(E_{0}\right)}-\left(\overline{D_{o_{s f}}\left(E_{0}\right)}\right)^{2}}{\left(\overline{D_{o_{s f}}\left(E_{0}\right)}\right)^{2}}\right]^{1 / 2}=\left[\frac{2}{d^{\prime}} \frac{\overline{M_{0}^{2}\left(E_{0}\right)}-\left(\overline{M_{0}\left(E_{0}\right)}\right)^{2}}{\left(\overline{M_{0}\left(E_{0}\right)}\right)^{2}}\right]^{1 / 2},
$$

where

$$
\begin{aligned}
& \overline{M_{0}^{2}\left(E_{0}\right)}=\overline{\left\langle E_{0}\left|\mathcal{O}^{T} \mathcal{O}\right| E_{0}\right\rangle^{2}}=\overline{\left[\sum_{\nu}\left\langle E_{\nu}|\mathcal{O}| E_{0}\right\rangle\right]^{2}} \\
& =\sum_{\nu} \overline{\left\langle E_{\nu}|\mathcal{O}| E_{0}\right\rangle^{4}}+\sum_{\nu \neq \mu} \overline{\left\langle E_{\nu}|\mathcal{O}| E_{0}\right\rangle^{2}} \overline{\left\langle E_{\nu}|\mathcal{O}| E_{0}\right\rangle^{2}} \\
& =\sum_{\nu}\left[\overline{\left\langle E_{\nu}|\mathcal{O}| E_{0}\right\rangle^{4}}-\left(\overline{\left\langle E_{\nu}|\mathcal{O}| E_{0}\right\rangle^{2}}\right)^{2}\right]+\left(\overline{M_{0}\left(E_{0}\right)}\right)^{2} .
\end{aligned}
$$

The step from the first to the second line in the previous expression follows from the independence of the strengths due to the P-T assumption, and the third line is obtained by adding and subtracting terms with $E_{\nu}=E_{\mu}$. Using again that $\overline{\hat{R}^{2}}=3$, this result can be simplified as

$$
\overline{M_{0}^{2}\left(E_{0}\right)}=2 \sum_{\mu}\left(\overline{\left\langle E_{\nu}|\mathcal{O}| E_{0}\right\rangle^{2}}\right)^{2}+\left(\overline{M_{0}\left(E_{0}\right)}\right)^{2} .
$$

This expression was reported for the first time in [59] (see also [40]), and its predictions have been compared with shell model results in [40, 60] for the width of the strength sums fluctuations. Finally, inserting (26) into (23), one gets

$$
\Delta_{o_{s f}}^{t y p}=\left[\frac{4}{d^{\prime}} \frac{\sum_{\mu}\left(\overline{\left\langle E_{\nu}|\mathcal{O}| E_{0}\right\rangle^{2}}\right)^{2}}{\left(\overline{M_{0}\left(E_{0}\right)}\right)^{2}}\right]^{1 / 2}=\left[\frac{4}{3 d^{\prime}}\right]^{1 / 2}\left({\overline{\mathrm{NPC}_{E_{0}}^{\mathrm{EGOE}}}}^{-1 / 2}\right.
$$

which establish a connection between the thermalization of the system, relative to an observable $\mathcal{O}_{s f}=\mathcal{O}^{T}(1) \mathcal{O}(1)$, and the value of the NPC for the transition strengths generated by $\mathcal{O}(1)$ acting on the eigenstate with energy $E_{0}$. An important outcome is that for chaotic systems the NPC is expected to be large and hence these system will thermalize, while for regular systems NPC has to be small and thus thermalization will be hindered.

It is also possible to derive an expression for generic operators when $\lambda>\lambda_{t}$. In this case the eigenstates become more and more delocalized in the mean-field basis. Finally we reach a regime were they behave as (quasi) random combinations of the basis states, the squared amplitudes $\left|U_{K}\right|^{2}$ abide the P-T distribution and $S^{\text {inf }}=S_{G O E}^{\text {inf }}\left(R^{\text {inf }}=1\right)$. In this regime the expression (16), valid for a generic observable, and (27) for $\mathcal{O}_{s f}$, take on a very simple form. Using Eq. (17) to expand $\left|E_{0}\right\rangle$ in the mean-field basis, one gets 


$$
\begin{aligned}
& \overline{D_{o}\left(E_{0}\right)} \stackrel{R^{i n f}}{\Longrightarrow}{ }^{d} \sum_{K=1}^{d} \overline{U_{K}^{2}}\langle K|\mathcal{O}| K\rangle=\frac{1}{d} \sum_{K=1}^{d}\langle K|\mathcal{O}| K\rangle=\langle\mathcal{O}\rangle, \\
& \overline{D_{o}\left(E_{0}\right)^{2}} \stackrel{R^{i n f} \rightarrow 1}{\Longrightarrow}\langle\mathcal{O}\rangle^{2}+\sum_{K=1}^{d} \overline{U_{K}^{4}-3 U_{K}^{2}}|\langle K|\mathcal{O}| K\rangle|^{2} \\
&+2 \sum_{K, K^{\prime}=1}^{d} \overline{U_{K}^{2}} \overline{U_{K^{\prime}}^{2}}\left|\left\langle K|\mathcal{O}| K^{\prime}\right\rangle\right|^{2}=\frac{2}{d}\left\langle\mathcal{O}^{2}\right\rangle+\langle\mathcal{O}\rangle^{2},
\end{aligned}
$$

where the usual P-T formulas for the moments, $\overline{\left|U_{K}\right|^{2}}=1 / d$ and $\overline{\left|U_{K}\right|^{4}}=3 / d^{2}$, have been applied.

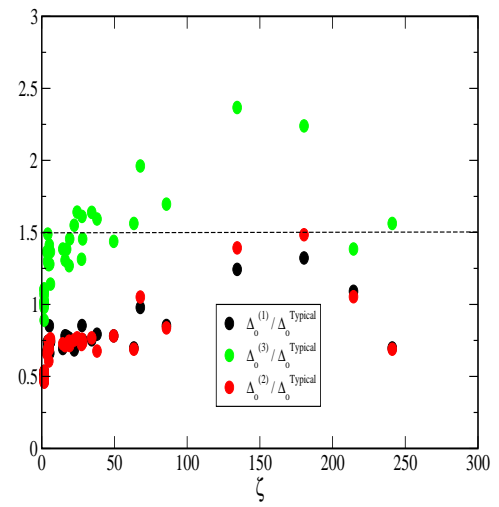

Figure 8. $\overline{\Delta_{o}} / \Delta_{o}^{t y p}$ values for $\mathcal{O}=\mathcal{O}_{d}(1), \mathcal{O}(1), \mathcal{O}(2)$ and $\mathcal{O}_{s f}$ in 60 member EGOE $(1+2)$ systems with $m=5,6$ and $n=12-16$, prepared at $t=0$ in initial states $\Psi(0)=\Psi^{(1)}(0)$ (black circles), $\Psi^{(2)}(0)$ (red squares) and $\Psi^{(3)}(0)$ (green diamonds), with energy $E \approx 0$.

Gathering these expressions together with (16) one finally arrives to

$$
\Delta_{o}^{t y p}=\left[\frac{4}{d d^{\prime}} \frac{\left\langle\mathcal{O}^{2}\right\rangle}{\langle\mathcal{O}\rangle^{2}}\right]^{1 / 2},
$$

that links the value of $\Delta_{o}^{t y p}$ with the dimension $d$ of the whole Hilbert space, the dimension $d^{\prime}$ of the microcanonical energy shell and the correlations generated by the operator $\mathcal{O}$, which are measured here by the ratio $\zeta=\left[\left\langle\mathcal{O}^{2}\right\rangle /\langle\mathcal{O}\rangle^{2}\right]^{1 / 2}$. Fig. 8 displays the values of $\overline{\Delta_{o}} / \Delta_{o}^{t y p}$ calculated for the four observables $\mathcal{O}_{d}(1), \mathcal{O}(1), \mathcal{O}(2)$ and $\mathcal{O}_{s f}$ in EGOE $(1+2)$ systems with $m=5,6$ and $n=12-16$. These are prepared in three different initial states $\Psi(0)=\Psi^{(1)}(0), \Psi^{(2)}(0)$ and $\Psi^{(3)}(0)$, with energy $E \approx 0$. It is clearly seen that $0 \leq \overline{\Delta_{o}} / \Delta_{o}^{t y p} \leq 3$, confirming that $\Delta_{o}^{\text {typ }}$ really behaves as a standard deviation. 
Using propagation formulas for $\langle\mathcal{O}\rangle$ and $\left\langle\mathcal{O}^{2}\right\rangle$ in terms of $m$ and $n$ one can obtain particular expressions of $\Delta_{o}^{t y p}$ for the operators considered in this paper. For instance, following the results given in Appendix E of [40] we have

$$
\Delta_{o_{d}(1)}^{t y p} \sim\left[\frac{4}{d d^{\prime}}\left\{1+\frac{1}{m} \frac{\left\langle\theta^{2}\right\rangle}{\langle\theta\rangle^{2}}\right\}\right]^{1 / 2},
$$

for $O_{d}(1)$ in the so called dilute limit, i.e., $m, n \longrightarrow \infty, m / n \longrightarrow 0$. Similar expressions can be derived for the other operators.

An important outcome is the presence of the factor $\left(d d^{\prime}\right)^{-1 / 2}$. Since the energy window must be sufficiently narrow so that the state density is constant inside, but wide enough to contain a large number of states, we can assume that $d^{\prime}=d / x$, where $x$ is essentially a fixed number. For instance, in the energy shell $W_{1}$ we have $x \simeq 14$. This result suggest that $\Delta_{o}^{t y p}$ is inversely proportional to the size of Hilbert space. However it should be noted that this trend may be modified by sharp variations of the correlations measured by $\zeta$ (or $\left\langle\theta^{2}\right\rangle /\langle\theta\rangle^{2}$ ) and by the fluctuations of the actual value $\Delta_{o}$ with regard to $\Delta_{o}^{\text {typ }}$.

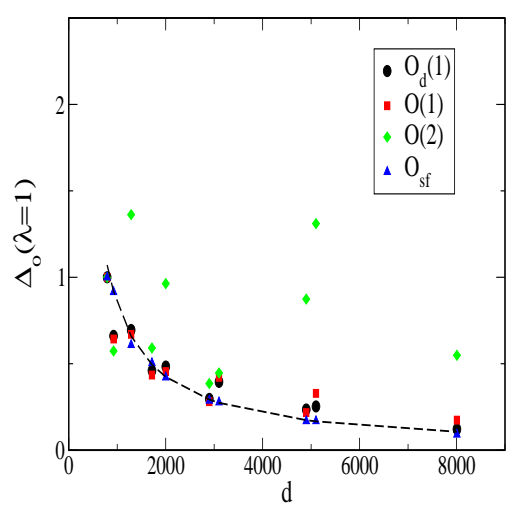

Figure 9. Evolution of the relative errors $\overline{\Delta_{o_{d}(1)}}, \overline{\Delta_{o(1)}}, \overline{\Delta_{o(2)}}$, and $\overline{\Delta_{o_{s f}}}$, calculated at $\lambda=1$, as the dimension of the Hilbert space increases. Their values have been rescaled so that $\overline{\Delta_{o}}=1$ for $d=792$. The dotted line stands for $\Delta_{o}=a / d$, where the value $a \approx 848$ has been obtained by a least-squares fit to the data.

Fig. 9 displays the evolution of $\overline{\Delta_{o}}$ at $\lambda=1$ as the dimension of the Hilbert space increases. The values of $\overline{\Delta_{o}}$ have been rescaled so that $\overline{\Delta_{o}}=1$ for $d=792$. In most cases the values of $\overline{\Delta_{o}}$ fall very close to the $\Delta_{o} \propto d^{-1}$ law, represented by the dotted line. The fluctuations of the relative error $\overline{\Delta_{o(2)}}$ are clearly larger due to larger oscillations of the corresponding $\zeta$ values. This fact is largely consistent with our previous findings because one expects the correlations of two-body operators to be more complex than those of onebody operators. In order to determine more precisely if the $d^{-1}$ is valid larger dimensions would be required, but this is a formidable computational task. Nevertheless, these results suggest that fully chaotic systems will thermalize relative to most observables in the thermodynamic limit. 


\section{Conclusions}

We have studied the thermalization process in isolated fermionic systems, described by EGOE $(1+2)$ ensembles. Our thermalization criterion relies on the ergodicity principle for the expectation of observables, which is more general than other criteria previously used, like the representability of occupancies by the Fermi-Dirac or Bose-Einstein distributions. Two-body random ensembles are paradigmatic models to study quantum chaos and the dynamical transition from integrability to chaos. It is well known that as the strength of the residual interaction is increased these systems undergo a order-tochaos transition, characterized by several chaos markers like $\lambda_{c}$, that signals the onset of GOE spectral fluctuations, and $\lambda_{t}$ which defines a region where different definitions of thermodynamic variables give essentially the same results, as it occurs for infinite systems. For $\lambda>\lambda_{t}$ the Hamiltonian eigenstates become more and more delocalized in the mean-field basis. We have shown by means of exact diagonalizations that the onset of Wigner spectral fluctuations is not sufficient to guarantee thermalization in finite systems. Only if all chaos signatures are fulfilled, including the quasi complete delocalization of eigenstates, we find that thermalization occurs for certain types of observables, such as (linear combinations of) occupancies and strength function operators.

As stated by many authors, the eigenstate thermalization hypothesis seems to be the mechanism responsible for thermalization, but in turn it holds only for certain observables provided that the eigenstates behave as quasi-random superpositions of basis states. We have also analyzed the influence of other factors, and found that the proximity of the initial state to the spectrum edges hinders thermalization. On the contrary large Hilbert space dimensions and initial states with a very small energy incertitude, i.e., with a very narrow distribution in the energy eigenbasis, enhance the thermalization process.

Analytical expressions linking the degree of thermalization for a given observable $\mathcal{O}$ with different properties of the system have been deduced. For instance, we have found that the typical value of the relative error between the equilibrium and microcanonical averages, $\Delta_{o}^{t y p}$, is inversely proportional to the square root of the NPC (or IPR) for transition strengths generated by $\mathcal{O}$ acting on the middle of the microcanonical shell. An important outcome of this result is that for chaotic systems the NPC is expected to be large and hence these system will thermalize, while for regular systems NPC has to be small and thus thermalization will be hindered.

Similarly, we have found that when the eigenstates become fully delocalized, $\Delta_{0}^{t y p}$ is proportional to the square root of the correlations generated by the observable and inversely proportional to the square root of the dimension $d$ of the whole Hilbert space times the dimension $d^{\prime}$ of the microcanonical energy shell. Since $d^{\prime} \propto d$, this result

shows that $\Delta_{0}^{t y p} \propto d^{-1}$ and suggests that fully chaotic systems will thermalize relative to most observables in the thermodynamic limit.

In conclusion we have presented the first study of thermalization in the two-body 
random matrix ensembles using the ergodicity principle for the expectation values of observables.

\section{Acknowledgments}

The authors A. R. and J. R. are thankful to L. Muñoz and J. M. G. Gómez for their collaboration and enlightening discussions. V. K. B. K. and M. V. thank Navinder Singh for useful discussions. This work is supported in part by Spanish Government grants for the research projects FIS2006-12783-C03-02, FIS2009-11621-C02-01, CSPD-2007-00042Ingenio2010, and by the Universidad Complutense de Madrid grant UCM-910059. One of us, A. R., is supported by the spanish program JAE-Doc.

\section{References}

[1] L. E. Reichel, A Modern Course in Statistical Physics, University of Texas, Austin (1980).

[2] Jördens R et al 2008 Nature 455204.

[3] Schneider U et al 2008 Science $\mathbf{3 2 2} 1520$

[4] Perfetti L et al 2006 Phys. Rev. Lett. 97067402

[5] Konishita T, Wenger T and Weiss D S 2006 Nature 440900

[6] Hofferberth S, Lesanovsky I, Fisher B, Schumm T and Schmiedmayer J 2007 Nature 449324

[7] Zhang W M and Feng D H 1995 Phys. Rep. 2521

[8] Relaño A, Dukelsky J, Gómez J M G and Retamosa J 2004, Phys. Rev. E 70026208

[9] Stöckmann H-J (1999) Quantum Chaos (Cambridge: Cambridge University Press)

[10] Haake F 2010 Quantum Signatures of Chaos (Heidelberg: Springer-Verlag)

[11] Iglói F and Rieger H 2000 Phys. Rev. Lett. 853233

[12] Cazalilla M A 2006 Phys. Rev. Lett. 97156403

[13] Calabrese P and Cardy J 2006 Phys. Rev. Lett. 96136801

[14] Sengupta K, Powell S and Sachdev S 2004 Phys. Rev. A 69053616

[15] Cherng R W and Levitov L S 2006 Phys. Rev. A 73043614

[16] Tasaki H 1998 Phys. Rev. Lett. 801373

[17] Linden N, Popescu S, Short A J and Winter A 2009 Phys Rev. E 79061103

[18] Reimann P 2008 Phys. Rev. Lett. 101190403

[19] Goldstein S, Lebowitz J L, Mastrodonato C, Tumulka R and Zanghi N 2010 Phys. Rev. E 81 011109

[20] Kollath C, Läuchli A M and Altman E 2007 Phys. Rev. Lett. 98180601

[21] Manmana S R, Wessel S, Noack R M and Muramatsu A 2007 Phys. Rev. Lett. 98210405

[22] Rigol M, Dunjko V, Yurovsky V and Olshanii M 2008 Nature 452854

[23] Rigol M 2009 Phys. Rev. Lett. 103100403

[24] Rigol M 2009 Phys. Rev. A 80053607

[25] Deutsch J M 1991 Phys. Rev. A 43 2046; arXiv:0911:0056

[26] Srednicki M 1994 Phys. Rev. E 50888

[27] Berry M V 1977 J. Phys. A 102083

[28] Santos L F and Rigol M 2010 Phys. Rev. E 81036206

[29] Relaño A 2010 J. Stat. Mech. P07016

[30] Olshanii M and Yurovsky V 2009 arXiv:0911.5587

[31] Neuenhahn C and Marquardt F 2010 arXiv:1007.5306

[32] J. B. French 1980 Moment Methods in Many Fermion Systems, (New York: Plenum)

[33] Horoi M, Zelevinsky V and Brown B A 1995 Phys. Rev. Lett. 745194

[34] Kota V K B and Sahu R 2002 Phys. Rev. E 66037103 
[35] Brody T A, Flores J, French J B, Mello P A, Pandey A and Wong S S M 1981 Rev. Mod. Phys. 53385

[36] Borgonovi F, Guarneri I, Izrailev F M and Casati G 1998 Phys. Lett. A 247140

[37] Flambaum V V, Izrailev F M and Casati G 1996 Phys. Rev. E 542136

[38] Flambaum V V and Izrailev F M 1997 Phys. Rev. E 565144

[39] Benenti G, Casati G and Shepelyansky D L, 2001 Euro. Phys. J. D 17265

[40] Kota V K B 2001 Phys. Rep. 347223

[41] Gómez J M G, Kar K, Kota V K B, Molina R A, Relaño A and Retamosa J 2011 Phys. Rep. 499 103

[42] Berry M V and Tabor M 1977 Proc. R. Soc. London A 356375

[43] Bohigas O, Giannoni M J and Schmit C 1984 Phys. Rev. Lett. 521

[44] Mehta M L 2004 Random Matrices (Amsterdam: Elsevier)

[45] Heusler S, Müller S, Altland A, Braun P and Haake F 2007 Phys. Rev. Lett. 98044103

[46] Vyas Manan, Kota V K B and Chavda N D 2010 Phys. Rev. E 81036212

[47] Angom D, Ghosh S and Kota V K B 2004 Phys. Rev. E 70016209

[48] Åberg S 1990 Phys. Rev. Lett. 643119

[49] Åberg S 1992 Prog. Part. Nucl. Phys. 2811

[50] Jacquod Ph and Varga I 2002 Phys. Rev. Lett. 89134101

[51] Jacquod Ph and Shepelyansky D L 1997 Phys. Rev. Lett. 791837

[52] It is also worth noting here that the actual value of the Brody parameter for GOE is not $\omega=1$, but $\omega=0.965$.

[53] Sakhr J and Nieminen J M 2005 Phys. Rev. E 72 045204(R)

[54] Berkovits R and Avishai Y 1998 Phys. Rev. Lett. 80568

[55] Kota V K B and Sahu R 2001 Phys. Rev. E 64016219

[56] Kota V K B 2003 Ann. Phys. (N.Y.) 30658

[57] Kota V K B and Haq R U 2010 Spectral Distributions in Nuclei and Statistical Spectroscopy (Singapore: World Scientific)

[58] Santos L F and Rigol M 2010 Phys. Rev. E 82031130

[59] Draayer J P, French J B and Wong S S M 1977 Ann. Phys. (N.Y.) 106472

[60] Gómez J M G, Kar K, Kota V K B, Molina R A and Retamosa J 2004 Phys. Rev. C 69057302

[61] Kota V K B and Sahu R 1998 Phys. Lett. B 4291 